\title{
Desarrollo de una aplicación móvil para el prediagnóstico y atención del VIH/SIDA.
}

\author{
Gisella Borja Roncallo \& Jesús Blanco Jiménez \\ Ingeniería Electrónica y Telecomunicaciones, Universidad Autónoma del Caribe, Barranquilla, Colombia. \\ gissella.borja25@uac.edu.co,jblancoj@cisco.com
}

Recibido: Febrero 23, 2020.

Recibido en su versión corregida: Abril 16, 2021.

https://doi.org/10.54606/Sextante2021.v24.03

Aceptación: Mayo 10, 2021.

Cómo citar: Borja Roncallo, G. \& Blanco Jiménez, J. (2021). Desarrollo de una aplicación móvil para el prediagnóstico y atención del VIH/SIDA. Revista Sextante, 24, pp. $20-28,2021$.

\section{Resumen}

La presente investigación expone el desarrollo de una aplicación móvil desde una perspectiva ingenieril, fundamentada en la línea de procedimiento de diseño y prototipado, basado en pantallas de interacción con el usuario para dar solución a un problema específico en el ámbito de la bioingeniería, la prevención por infección de VIH/SIDA por medio de la obtención de informes interpretativos, que son el resultado de las pruebas diagnósticas. Ergo, se recurre al diseño iterativo como método para la construcción de un prototipo funcional de una aplicación, titulada PreVIH, utilizando HTML/CCS/JS/WebSQL para ser instalada en teléfonos inteligentes que cuenten con el sistema operativo Android 9 o versiones posteriores. La app resultante demostró la practicidad de utilizar aplicaciones móviles en entornos donde la alta tecnología es de difícil acceso y la necesidad de tener al alcance información es fundamental para la atención primaria, donde los médicos tratantes no cuentan con especializaciones. De allí que, el enfoque de PreVIH sea viable para su mejora y posterior implantación.

Palabras clave: Aplicación móvil, App, Prediagnóstico, SIDA, VIH.

\section{Development of a mobile application for HIV/AIDS pre-diagnosis and care.}

\begin{abstract}
The present research exposes the development of a mobile application from an engineering perspective founded on the design and prototyping procedure line based on user-interactive screens to solve a specific issue in bioengineering, the prevention of HIV/AIDS infection employing medical examinations that are the result of diagnostic tests. Therefore, iterative design is used to build a functional prototype of an app, which is denominated PreVIH. It is written in HTML/CCS/JS/ WebSQL to be downloaded on smartphones with Android 9 or with later releases. The resulting app demonstrated the practicality of using mobile apps in environments where high technology is difficult to access; in places where approaching information is essential for primary care where treating physicians do not have specializations. Hence, the PreVIH approach is viable for its improvement and subsequent implementation.
\end{abstract}

Keywords: AIDS, App, HIV, Mobile app, Prototype. 


\section{Introducción}

En la actualidad el concepto de movilidad, aplicado a la salud, significa poder acceder a información optimizada para su lectura en pantallas pequeñas, en cualquier momento y lugar, gracias a los dispositivos móviles mejor conocidos como smartphones. Esto se traduce en un menor consumo de recursos y menos esfuerzo por parte del usuario. Tales ventajas son el fundamento para el desarrollo de un prototipo de aplicación móvil orientado a la prevención, que permita el prediagnóstico del Virus de Inmunodeficiencia Humana y el Síndrome de Inmunodeficiencia Adquirida (VIH/SIDA) en aquellas zonas menos favorecidas, donde no se cuenta con laboratorios especializados 0 especialistas en VIH/SIDA que puedan emitir un diagnóstico interpretativo.

Para ello, la propuesta ingenieril se realiza a partir del diseño iterativo basado en la experiencia del usuario (UX) y la interfaz de usuario (UI); es decir, en las reglas y el comportamiento de la aplicación móvil para satisfacer las necesidades de información y retroalimentación, de acuerdo con el procedimiento establecido en el algoritmo de diagnóstico de VIH en adultos, emitido por el Ministerio de Salud y Protección Social (MSPS) de Colombia.

Por último, es preciso mencionar las limitantes encontradas para acceder a resultados de pruebas diagnósticas por infección VIH/SIDA y así poder alimentar la aplicación, con el objetivo de realizar las diferentes pruebas funcionales y validar los resultados esperados; lo mismo obedece a la sensibilidad de los pacientes a compartir información. En consecuencia, se recurrió a obtener resultados de pruebas diagnósticas a través de búsquedas en la Internet.

\section{Metodología}

Según los propósitos de la investigación, la estrategia es de tipo aplicada, debido a que está orientada a desarrollar el prototipo de una aplicación móvil para el prediagnóstico del Virus de Inmunodeficiencia Humana y el Síndrome de Inmunodeficiencia Adquirida (VIH/SIDA). Para el desarrollo de esta aplicación móvil se siguieron las bases del diseño iterativo junto a las etapas del desarrollo de aplicaciones móviles, lo cual va más allá del desarrollo de un software tradicional. Estas son: conceptualización, definición, diseño, desarrollo y publicación.

En la fase de conceptualización se formaliza la idea de la aplicación móvil, teniendo en cuentas las necesidades de los usuarios directos e indirectos. Posteriormente, se define el alcance del proyecto, la complejidad de la programación a través de los requerimientos funcionales o tareas que debe realizar la aplicación.

A continuación, en la fase de diseño se definen los escenarios, estructura de la aplicación web a través de diagramas; también la Interfaz Gráfica de Usuario (IGU) a través de wireframe y mockups, pasando de un prototipo de baja fidelidad a uno de alta fidelidad, a fin de validar el aspecto visual. Seguidamente, en la etapa de desarrollo se codifica la aplicación móvil haciendo uso de HTML5/CCS/JS y la API Web SQL Database, se documenta y realizan pruebas unitarias para probar el correcto funcionamiento de los elementos programados y depurar los errores encontrados. Por último, se generará un archivo apk para que los usuarios puedan instalarlo en un equipo móvil con sistema Android 9 o superior.

\section{Análisis de resultados y propuesta ingenieril}

PreVIH[App] es el nombre del prototipo de aplicación móvil desarrollado para el prediagnóstico del VIH/SIDA. Su objetivo es permitir al personal médico de atención primaria, que se ubica en entornos sanitarios menos complejos, el registro de los resultados de las PR para el diagnóstico por infección de VIH/SIDA, practicadas a hombres y mujeres (no gestantes), mayores de 18 años, y obtener una interpretación de los resultados, así como, las medidas e indicaciones a seguir.

\subsection{Requerimientos de la aplicación móvil}

A continuación, en la Tabla 1, se especifican las características operacionales de la aplicación móvil y se establecen las restricciones que debe cumplir.

\subsection{Modelado del usuario}

En este apartado se presenta el arquetipo de la persona (ver Figura 1) que será el usuario focal de la aplicación móvil. Posteriormente, para poder con- 
Tabla 1. Requerimientos de la aplicación móvil.

\begin{tabular}{ll}
\hline Tipo de requerimiento & \multicolumn{1}{c}{ Descripción } \\
\hline Ambiente físico & $\begin{array}{l}\text { La app necesita para funcionar un equipo móvil } \\
\text { o tableta. } \\
\text { La app es híbrida. } \\
\text { Para utilizar la app se necesitará el sistema } \\
\text { operativo Android 9 o versiones superiores }\end{array}$ \\
\hline Interfaz & $\begin{array}{l}\text { Las entradas de información provienen de los } \\
\text { usuarios. }\end{array}$ \\
& La aplicación HIVSmart sirve de referencia. \\
\hline Usuarios & $\begin{array}{l}\text { La app será utilizada por médicos/especialistas. } \\
\text { El tipo de usuario será único focal. }\end{array}$ \\
\hline Funcionalidad & $\begin{array}{l}\text { El usuario debe saber manejar dispositivos } \\
\text { móviles. }\end{array}$ \\
& $\begin{array}{l}\text { Registro e interpretación de los resultados de } \\
\text { pruebas diagnósticas por infección VIH/SIDA. }\end{array}$ \\
\hline Recursos & $\begin{array}{l}\text { Para el diseño de la aplicación se requiere un } \\
\text { desarrollado de entornos HTML5/CCS/JS. El }\end{array}$ \\
tiempo de ejecución estimado es de 152 horas de \\
desarrollo.
\end{tabular}

Fuente: Los autores.

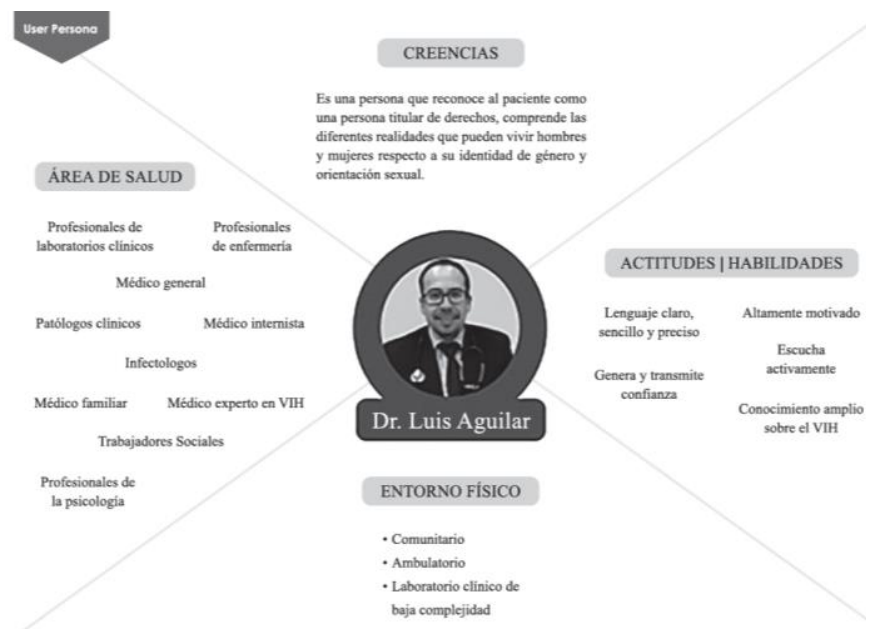

Figura 1. Arquetipo de usuario para la aplicación móvil. Fuente: Los autores.

-textualizar el proceso de interacción entre la persona y la aplicación se establecen los escenarios y los casos de uso.

\subsection{Definición de escenarios y casos de uso}

\section{El prototipo se compone de tres escenarios:}

Escenario F1: Cuando al establecimiento de salud llega una persona que quiere conocer su estado de salud frente al VIH, es mayor de edad y desea hacerlo de forma voluntaria. Se procede a realizarse la PR ELISA, que no requiere autorización médica, y al tener los resultados el médico/especialista debe interpretarlos. Para ello debe iniciar la aplicación móvil, iniciar sesión, registrar los datos del paciente y los resultados de la prueba presuntiva.

Escenario F2: Dado el resultado reactivo de la prueba presuntiva, el médico/especialista ordena una segunda prueba duplicada PR ELISA al paciente. Al tener los resultados el médico/especialista debe interpretarlos. Para ello, debe iniciar la aplicación móvil, iniciar sesión, buscar el paciente, registrar los resultados de esta prueba para obtener la interpretación, considerando los resultados obtenidos en la prueba presuntiva.

Escenario F3: Dado el resultado no reactivo de la segunda prueba, el médico/especialista ordena una tercera prueba confirmatoria Western Blot al paciente. Al tener los resultados el médico/especialista debe interpretarlos. Para ello, debe iniciar la aplicación móvil, iniciar sesión, buscar el paciente, registrar los resultados de esta prueba para obtener la interpretación considerando los resultados obtenidos en la prueba presuntiva y la prueba duplicada.

Lo anterior permite identificar seis casos de uso donde se describen narrativamente las diferentes interacciones entre el medico/especialista y la aplicación móvil: CU-0 Registro de pruebas diagnósticas (ver Tabla 2), CU-1 Iniciar sesión (ver Tabla 3), CU-1-1 Registro de usuario (ver Tabla 4), CU-2 Registro de paciente (ver Tabla 5), CU-2-1 Búsqueda de paciente (ver Tabla 6) y CU-3 Registro de prueba diagnóstica (ver Tabla 7).

Tabla 2. Caso de uso registro de pruebas diagnósticas.

\begin{tabular}{ll}
\hline \multicolumn{1}{c}{ ID y Nombre } & \multicolumn{1}{c}{ CU-0 Registro de pruebas diagnósticas } \\
\hline Fecha de creación & $15 / 05 / 2021$ \\
\hline Actores principales & Médico/Especialista. \\
\hline Actores secundarios & Base de datos. \\
\hline Descripción & $\begin{array}{l}\text { Registrar los resultados de las pruebas } \\
\text { diagnósticas realizadas en pacientes por } \\
\text { infección de VIH/SIDA. }\end{array}$ \\
\hline Precondiciones & $\begin{array}{l}\text { PRE-1: El usuario debe estar registrado en la app } \\
\text { y haber iniciado sesión. }\end{array}$ \\
\hline Postcondiciones & $\begin{array}{l}\text { POST-1: Prueba diagnóstica registrada en la base } \\
\text { de datos. }\end{array}$ \\
\hline Secuencia normal & $\begin{array}{l}\text { 1. El usuario abre la app. } \\
\text { 2. El sistema solicita los datos para iniciar sesión } \\
\text { [CU-1]. }\end{array}$ \\
$\begin{array}{l}\text { 3. El usuario registra al paciente [CU-2]. } \\
\text { 4. El usuario registra los resultados de la prueba } \\
\text { diagnóstica [CU-3]. } \\
\text { 5. La app muestra el análisis de resultados de la } \\
\text { prueba diagnóstica. }\end{array}$ \\
\hline
\end{tabular}

Fuente: Los autores. 
Tabla 3. Caso de uso inicio de sesión.

\begin{tabular}{ll}
\hline \multicolumn{1}{c}{ ID y Nombre } & \multicolumn{1}{c}{ CU-1 Iniciar sesión } \\
\hline Fecha de creación & $15 / 05 / 2021$ \\
\hline Actores principales & Médico/Especialista. \\
\hline Actores secundarios & Base de datos. \\
\hline Descripción & Permite que un usuario registrado ingrese en \\
& PreVIH[App]. \\
\hline Precondiciones & PRE-1: El usuario debe estar registrado en la \\
& base de datos. \\
\hline Postcondiciones & POST-1: El usuario ingresa en \\
& PreVIH[App]. \\
\hline Secuencia normal & 1. El usuario digita su CC y contraseña. \\
& 2. El usuario presiona "Listo". \\
& 3. La app valida los datos ingresados por el \\
& usuario (ver 2). \\
& 4. La app busca el usuario en la base de datos \\
& (ver 3). \\
\hline Excepciones & El usuario ingresa en la app. \\
& 3. Si los datos ingresados por el usuario no \\
& cumplen las restricciones impuestas por la \\
aplicación. & 3.1 La app notifica el error. \\
4. Si el usuario no se encuentra registrado en \\
la base de datos \\
4.1 La app notifica el error. \\
$\begin{array}{l}\text { 4.2 Se cancela el proceso de inicio de sesión. } \\
\text { 4.3 El usuario solicita a la app iniciar el } \\
\text { proceso para registrarse [CU-1-1]. }\end{array}$ \\
\end{tabular}

Fuente: Los autores.

Tabla 4. Caso de uso registro de usuario.

\begin{tabular}{ll}
\hline \multicolumn{1}{c}{ ID y Nombre } & \multicolumn{1}{c}{ CU-1-1 Registro de usuario } \\
\hline Fecha de creación & $15 / 05 / 2021$ \\
\hline Actores principales & Médico/Especialista. \\
\hline Actores secundarios & Base de datos. \\
\hline Descripción & $\begin{array}{l}\text { Permite registrar un nuevo usuario en } \\
\text { PreVIH[App]. }\end{array}$ \\
\hline Disparador & Al pulsar "Listo". \\
\hline Precondiciones & $\begin{array}{l}\text { PRE-1: El usuario no debe estar registrado } \\
\text { en la base de datos. }\end{array}$ \\
\hline Postcondiciones & $\begin{array}{l}\text { POST-1: El usuario se registra en la base de } \\
\text { datos. }\end{array}$ \\
\hline Secuencia normal &
\end{tabular}

1. El usuario pulsa "Registrar" para solicitar comenzar el proceso para el registro.

2. El usuario digita la CC, nombre, apellido, nombre de usuario, contraseña y verificación de contraseña.

3. El usuario presiona "Listo".

4. La app valida los datos ingresados por el usuario (ver 5).

5. La app busca al usuario en la base de datos (ver 6).

6. La app notifica que el usuario se ha registrado correctamente.

\begin{tabular}{ll}
\hline Excepciones & 5. Si los datos ingresados por el usuario no \\
cumplen las restricciones impuestas por la \\
aplicación. \\
5.1 La app notifica el error. \\
6. Si el usuario no se encuentra registrado en \\
la base de datos. \\
6.1 La app notifica el error. \\
6.2 Se cancela el proceso de registro de \\
usuario. \\
\hline
\end{tabular}

Fuente: Los autores.
Tabla 5. Caso de uso registro de paciente.

\begin{tabular}{|c|c|}
\hline ID y Nombre & CU-1 Iniciar sesión \\
\hline Fecha de creación & $15 / 05 / 2021$ \\
\hline Actores principales & Médico/Especialista. \\
\hline Actores secundarios & Base de datos. \\
\hline Descripción & $\begin{array}{l}\text { Permite registrar un nuevo usuario en } \\
\text { PreVIH[App]. }\end{array}$ \\
\hline Disparador & Al pulsar "Listo". \\
\hline Precondiciones & $\begin{array}{l}\text { PRE-1: El usuario debe estar registrado en la } \\
\text { app y haber iniciado sesión. } \\
\text { PRE-2: El paciente no debe estar registrado en } \\
\text { la base de datos. }\end{array}$ \\
\hline Postcondiciones & $\begin{array}{l}\text { POST-1: El paciente se registra en la base de } \\
\text { datos. }\end{array}$ \\
\hline Secuencia normal & $\begin{array}{l}\text { 1. El usuario pulsa "Agregar" para solicitar } \\
\text { comenzar el proceso para el registro. } \\
\text { 2. El usuario digita el CC, nombre, apellido, } \\
\text { número de contacto o WhatsApp, selecciona } \\
\text { sexo (femenino, masculino), identidad de } \\
\text { género (cisgénero, intersexual, transgénero, no } \\
\text { binario), orientación sexual (asexual, bisexual. } \\
\text { heterosexual, homosexual, pansexual), } \\
\text { selecciona factores de riesgo (ITS, hepatitis B, } \\
\text { hepatitis C, tuberculosis TB, alcoholismo, } \\
\text { drogadicción, lupus, cáncer), marca la casilla } \\
\text { de verificación "El paciente es mayor de edad } \\
\text { y accede de forma voluntaria a practicarse la } \\
\text { PR por infección de VIH/SIDA para conocer } \\
\text { su estado de salud". } \\
\text { 3. El usuario presiona "Listo". } \\
\text { 4. La app valida los datos ingresados por el } \\
\text { usuario (ver 4). } \\
\text { 5. La app busca el paciente en la base de datos } \\
\text { (ver 5). } \\
6 \text {. La app notifica que el paciente se ha } \\
\text { registrado correctamente. }\end{array}$ \\
\hline Excepciones & $\begin{array}{l}\text { 4. Si los datos ingresados por el usuario no } \\
\text { cumplen las restricciones impuestas por la } \\
\text { aplicación. } \\
\text { 4.1 La app notifica el error. } \\
5 \text {. Si el paciente se encuentra registrado en la } \\
\text { base de datos. } \\
5.1 \mathrm{La} \text { app notifica el error. } \\
5.2 \mathrm{Se} \text { cancela el proceso de registro de } \\
\text { paciente. }\end{array}$ \\
\hline
\end{tabular}

Fuente: Los autores.

Tabla 6. Caso de uso búsqueda de paciente.

\begin{tabular}{ll}
\hline \multicolumn{1}{c}{ ID y Nombre } & \multicolumn{1}{c}{ CU-2-1 Búsqueda de paciente } \\
\hline Fecha de creación & $15 / 05 / 2021$ \\
\hline Actores principales & Médico/Especialista. \\
\hline Actores secundarios & Base de datos. \\
\hline Descripción & $\begin{array}{l}\text { Buscar un paciente registrado en } \\
\text { PreVIH[App]. }\end{array}$ \\
\hline Disparador & $\begin{array}{l}\text { Al digitar la identificación del paciente en el } \\
\text { cuadro de búsqueda. }\end{array}$ \\
\hline Precondiciones & $\begin{array}{l}\text { PRE-1: El paciente tiene que estar almacenado } \\
\text { en la base de datos. }\end{array}$ \\
\hline Postcondiciones & \begin{tabular}{l} 
POST-1: El paciente se muestra en la lista. \\
\hline Secuencia normal
\end{tabular} \\
& $\begin{array}{l}\text { 1. El usuario pulsa el botón "Buscar". } \\
\text { paciente. }\end{array}$ \\
$\begin{array}{l}\text { 3. La app busca en la base de datos al paciente } \\
\text { (ver 3). }\end{array}$ \\
$\begin{array}{l}\text { 4. La app muestra en la lista al paciente. } \\
\end{array}$
\end{tabular}




\begin{tabular}{ll}
\hline Excepciones & 3. Si el paciente no se encuentra registrado en \\
la base de datos. & 3.1. La app notifica el error. \\
4. Se cancela el proceso de búsqueda del \\
paciente.
\end{tabular}

Fuente: Los autores.

Tabla 7. Caso de uso registro de prueba diagnóstica.

\begin{tabular}{ll}
\hline \multicolumn{1}{c}{ ID y Nombre } & \multicolumn{1}{c}{ CU-3 Registro de prueba diagnóstica } \\
\hline Fecha de creación & 15/05/2021 \\
\hline Actores principales & Médico/Especialista. \\
\hline Actores secundarios & Base de datos. \\
\hline Descripción & $\begin{array}{l}\text { Permite registrar en PreVIH[App] las pruebas } \\
\text { diagnósticas por infección VIH/SIDA } \\
\text { practicadas a pacientes hombres y mujeres, } \\
\text { mayores de 18 años, no gestantes. }\end{array}$ \\
\hline Disparador & Al pulsar "Listo". \\
\hline Precondiciones & $\begin{array}{l}\text { PRE-1: El usuario debe estar registrado en la } \\
\text { app y haber iniciado sesión. }\end{array}$ \\
\hline PRE-2: El paciente debe estar registrado en la \\
base de datos.
\end{tabular}

Fuente: Los autores.

\subsection{Arquitectura de la información}

La organización del contenido de la app se presenta en la Figura 2., de acuerdo a ésta, luego de haber ingresado al usuario, se encuentran tres opciones en la pantalla principal:
Opción 1. Pacientes. Tiene acceso a visualizar el listado de pacientes registrados, agregar un nuevo paciente y asociar factores de riesgo.

Opción 2. Pruebas. Tiene acceso a registrar las diferentes pruebas para el diagnóstico por infección de VIH/SIDA.

Opción 3. Análisis. Tiene acceso a visualizar el listado de pacientes registrados con pruebas prediagnósticas realizas y ver el informe de resultados.

\subsection{Wireframes de la aplicación móvil}

La representación simple de las pantallas se realiza en wireframes (ver Figuras 3 y 4 ) para mostrar la idea inicial de organización de los elementos, centrado en la estructura.

En esta primera aproximación se presenta un esbozo de la organización de los elementos, que contendrá: una primera evaluación de los aspectos, como navegación e interacción, los cuales dieron paso al diseño visual.

\subsection{Mockups de la aplicación móvil}

La maqueta de diseño digital de la app propuesta se realizó incluyendo los elementos de diseño en detalle de forma estática, integrando color e imágenes (ver Figuras 5 y $\underline{6}$ ).

\subsection{Estructura de la base de datos}

La base de datos consta de cuatro tablas: TableUsuarios, TablePacientes, TableFactores y TablePruebas (ver Figura 7).

\subsection{Pruebas unitarias de funcionamiento}

Para evaluar la IU y UX se utilizaron los criterios de calidad básica para las apps publicado en Android Developers (2021), específicamente los relacionados con la experiencia visual y estabilidad. 


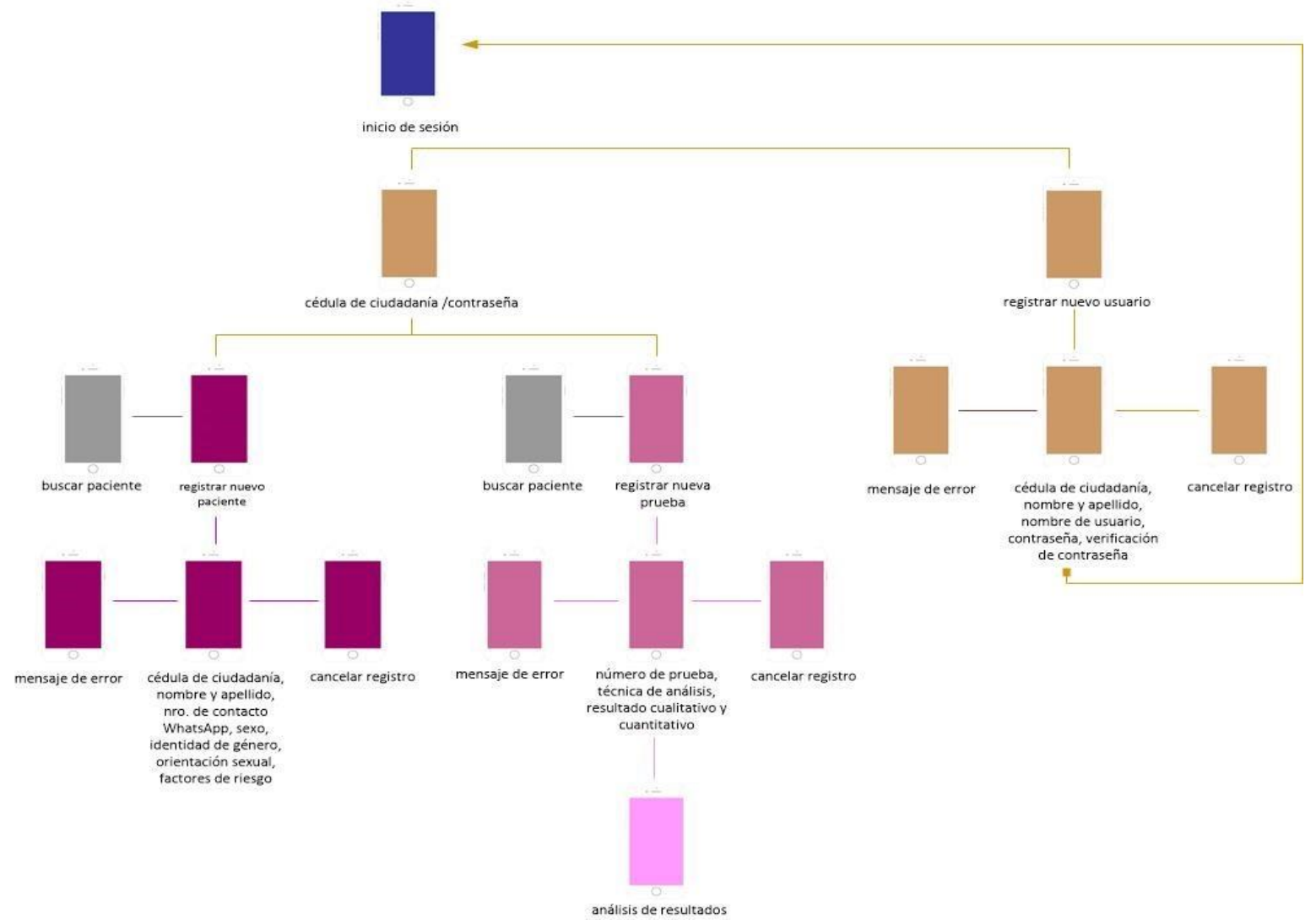

Figura 2. Diagrama de arquitectura de información de la aplicación móvil.

Fuente: Los autores.

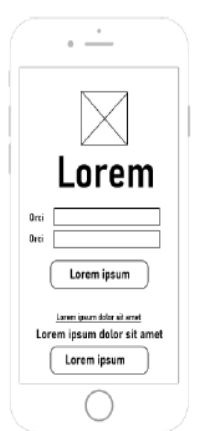

Pantalla Inicio de sesión

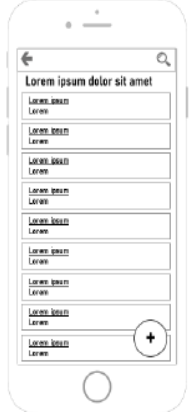

Pantalla Listado de pacientes

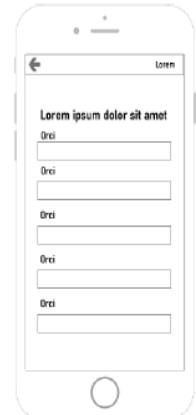

Pantalla Registro de usuario

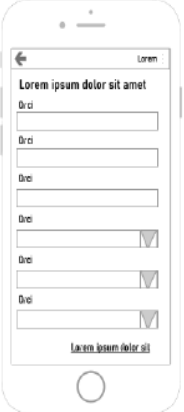

Pantalla Registro de paciente

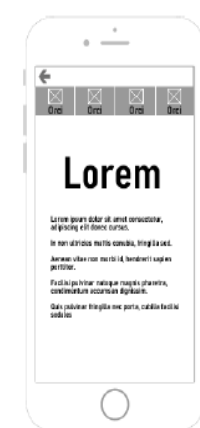

Pantalla Inicial/pestañas

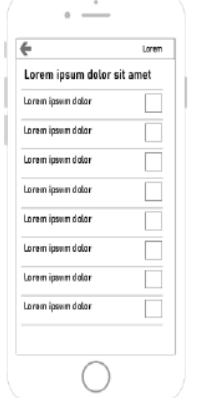

Pantalla Factores de riesgo
Figura 3. Wireframes 1-6 de la aplicación móvil. Fuente: Los autores.
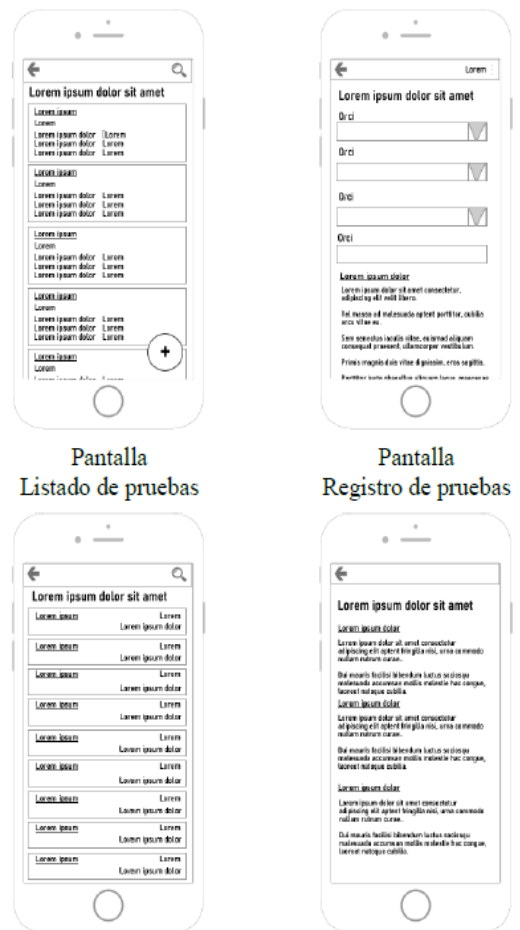

Pantalla Listado de resultados Registro de pruebas

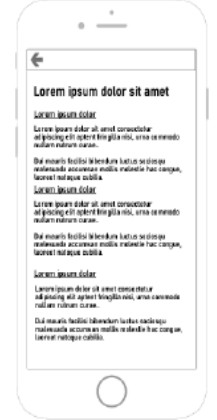

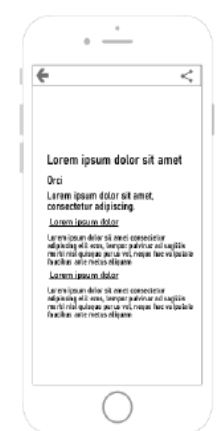

Pantalla Resultados de pruebas

Términos y condiciones

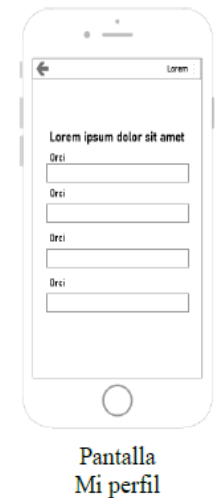

Figura 4. Wireframes 7-12 de la aplicación móvil.. Fuente: Los autores. 


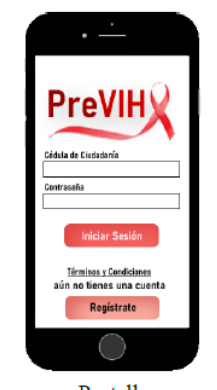

Pantalla Inicio de sesión

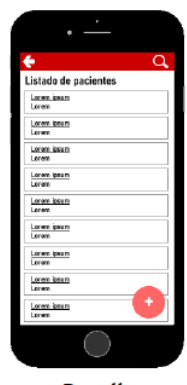

Pantalla

Listado de pacientes

Nota: elaboración propia

Figura 5. Mockups 1-6 de la aplicación móvil.

Fuente: Los autores.

\section{- Pruebas realizadas}

Prueba CR-3 desde cada una de las pantallas (y diálogos) de la app, se presiona el botón atrás o usa el gesto para deslizar hacia atrás.

Prueba CR-5 desde cada una de las pantallas de la app, se gira el dispositivo entre las orientaciones horizontal y vertical al menos tres veces.

Prueba CR-6 cambiar a otra app para enviar la que se está probando a segundo plano. Ingresar en configuración y comprobar si la app que se está probando tiene servicios ejecutándose mientras se encuentra en segundo plano.

\section{- Hallazgos de la app PreVIH}

Admite la navegación estándar del botón atrás.

No admite compatibilidad con la navegación por gestos.

Preserva el estado cuando pasa a segundo plano $y$ regresa con el estado exacto en que se encontraba al pasar nuevamente a primer plano.

No evita la pérdida accidental de datos a causa de la navegación hacia atrás.
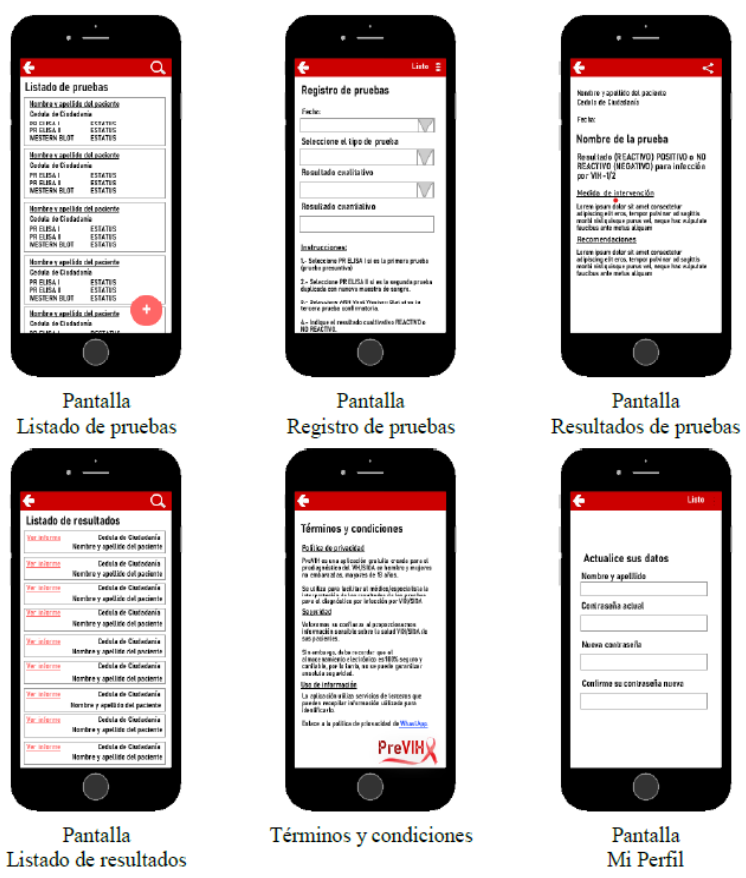

Figura 6. Mockups 7-12 de la aplicación móvil.

Fuente: Los autores.
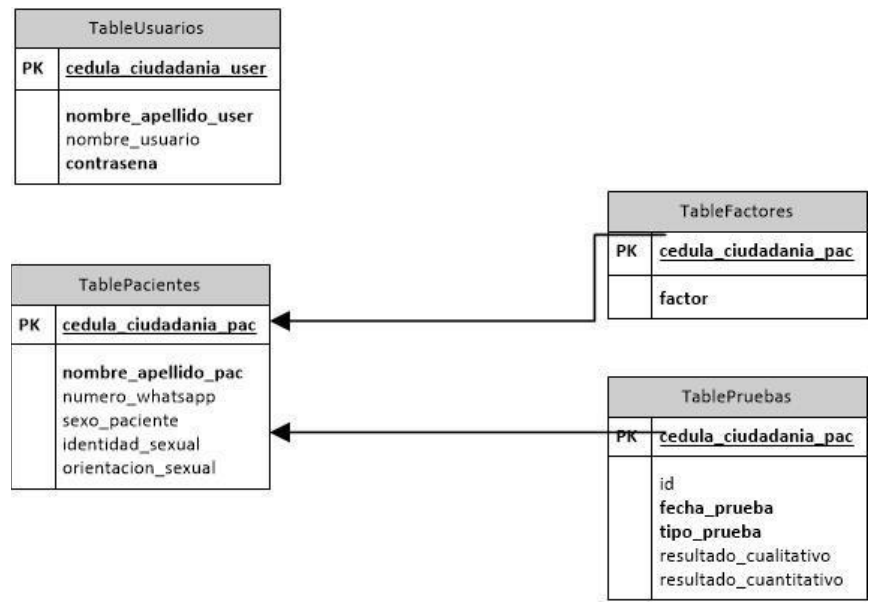

Figura 7. Estructura de la base de datos de la aplicación móvil.

Fuente: Los autores.

Admite tanto la orientación horizontal como la vertical.

No utiliza toda la pantalla en ambas orientaciones y no usa formato de pantalla ancha para compensar los cambios de orientación.

Muestra texto y bloques de texto de forma aceptable.

Se carga rápidamente.

Al ser instalada solicita solo la cantidad mínima absoluta de permisos (ver Figura 8). 
Evita que se ejecuten servicios en segundo plano (ver Figura 9).

Es estable, no falla ni bloquea el subproceso de UI que provoca errores de Android.

\section{Conclusiones}

Los métodos utilizados para el diagnóstico presuntivo, confirmatorio y precoz de la infección por VIH/SIDA en hombres y mujeres, mayores de 18 años, no gestantes, que se encuentran en el algoritmo de diagnóstico de VIH en adultos emitido por el MSPS de Colombia, fueron claves para determinar los requerimientos funcionales y no funcionales de la aplicación móvil, permitiendo establecer los diferentes escenarios y casos de uso a partir del contexto y los usuarios, directos e indirectos. Todo esto se confirma con los resultados obtenidos de las diferentes pruebas aplicadas para verificar su funcionalidad.

El diseño del prototipo de la aplicación móvil desarrollada recopila con eficacia los resultados de las pruebas diagnósticas presuntivas $\mathrm{y}$ confirmatorias, para emitir un prediagnóstico de la infección por VIH/SIDA en hombres y mujeres, mayores de 18 años, no gestantes; estableciendo siete casos de aplicación que arrojan informes interpretativos diversos, ajustados a cada caso en específico, incluyendo sus respectivas medidas de intervención.

Se valida la aplicación móvil para el prediagnóstico del VIH/SIDA en hombres y mujeres, mayores de 18 años, no gestantes, donde el personal médico esté capacitado en la atención primaria de la salud; considerándose útil pues se confirma que la aplicación puede emitir una interpretación de forma rápida y eficiente, sin requerir ser especialista en materia de VIH. Por lo tanto, su adaptabilidad a entornos donde no existan laboratorios especializados y especialistas del área es viable.

En definitiva, el desarrollo de aplicaciones móviles nativas basadas en la tecnología que ofrece Android es una herramienta que debe ser aprovechada, no solo en el ámbito de salud, sino en cualquier ambiente que carezca de recursos, a fin de mejorar el acceso a la información.

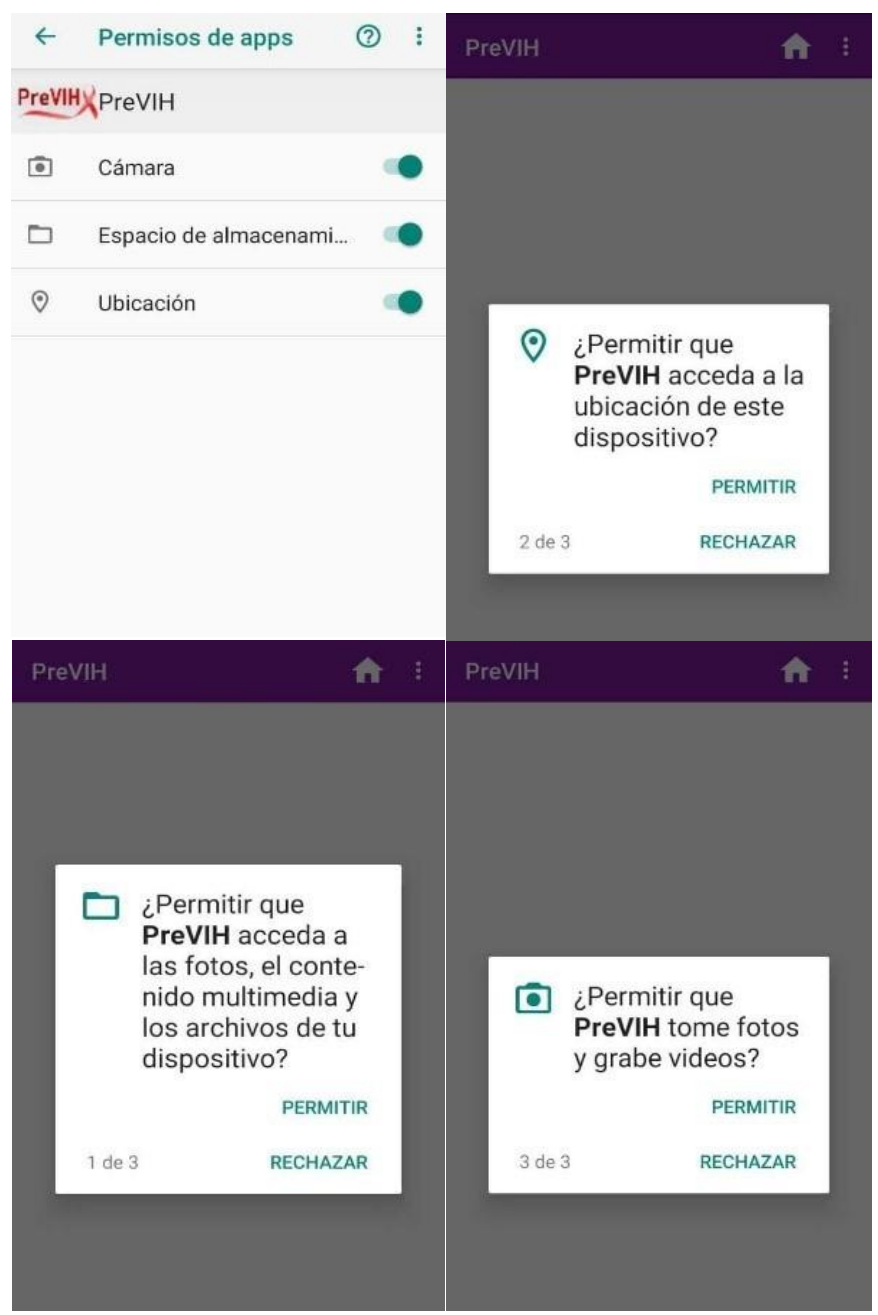

Figura 8. PreVIH. Permisos requeridos al instalar.

Fuente: Los autores.

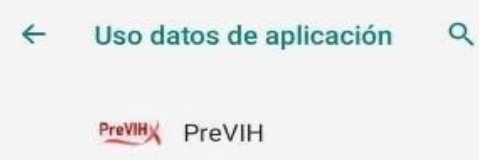

15 de may. -14 de jun.

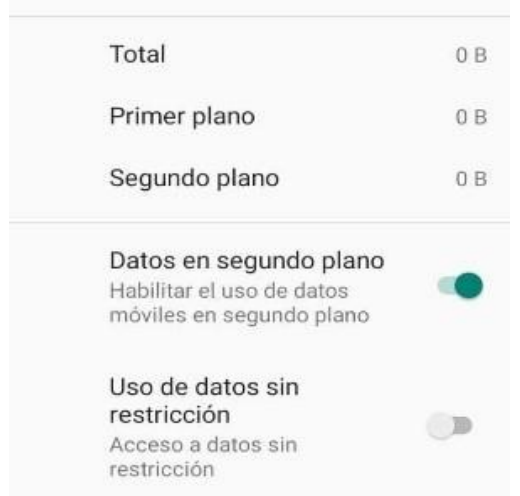

Figura 9. PreVIH. Servicios en segundo plano.

Fuente: Los autores. 


\section{Referencias}

Cho, H., Prorras, T.; Baik, D.; Beauchemin, M. y Schnall, R. (2018). Comprender los factores predisponentes, habilitadores y reforzantes que influyen en el uso de una aplicación de gestión del VIH basada en dispositivos móviles: una evaluación de usabilidad en el mundo real. Recuperado de: https://dx.doi.org/10.1016\%2Fj.ijmedinf.2018.0 $\underline{6.007}$

Hébuterne, S. (2018). Desarrolle una aplicación Android: programación en Java con Android Studio. Barcelona: ENI.

Luna, F. (2016). Desarrollo web para dispositivos móviles: herramientas para diseñar y programar WebApps. RedUsers.

Marescal, O. (2017). Investigador de McGill desarrolla una nueva aplicación de autoprueba del VIH HIVSmart!. Recuperado de: https://www.mcgilltribune.com/sci-tech/mcgillresearcher-develops-new-hiv-self-testing-apphivsmart-112717/

Ministerio de Salud y Protección Social. (2014). Guía de práctica clínica basada en la evidencia científica para la atención de la infección por VIH/Sida en adolescentes (con 13 años o más de edad) y adultos. Bogotá. Recuperado de: https://www.minsalud.gov.co/sites/rid/Lists/Bibl iotecaDigital/RIDE/VS/PP/GPC-corta-VIHADOLESCENTES-ADULTOS-final.pdf

Montero, R. (2014). Desarrollo de aplicaciones para Android. Madrid: RA-MA, S.A.

Serna, S. y Pardo, C. (2016). Diseño de interfaces en aplicaciones móviles. Madrid: RA-MA, S.A.

Schnall, R.; Bakken, S.; Rojas, M.; Travers, J. y Carballo, A. (2016). La tecnología mHealth como herramienta persuasiva para el tratamiento, la atención y la gestión de las personas que viven con el VIH. Recuperado de: https://dx.doi.org/10.1007\%2Fs10461-0140984-8

Schnall, R.; Patterson, J.; Iribarren, S.; Bakken, S.; Carballo, A. y Marrón, W. (2015). Comparación de una aplicación de autogestión y diseño centrado en el usuario con aplicaciones de salud móvil existentes para personas que viven con el VIH. Recuperado de: https://dx.doi.org/10.2196\%2Fmhealth.4882 